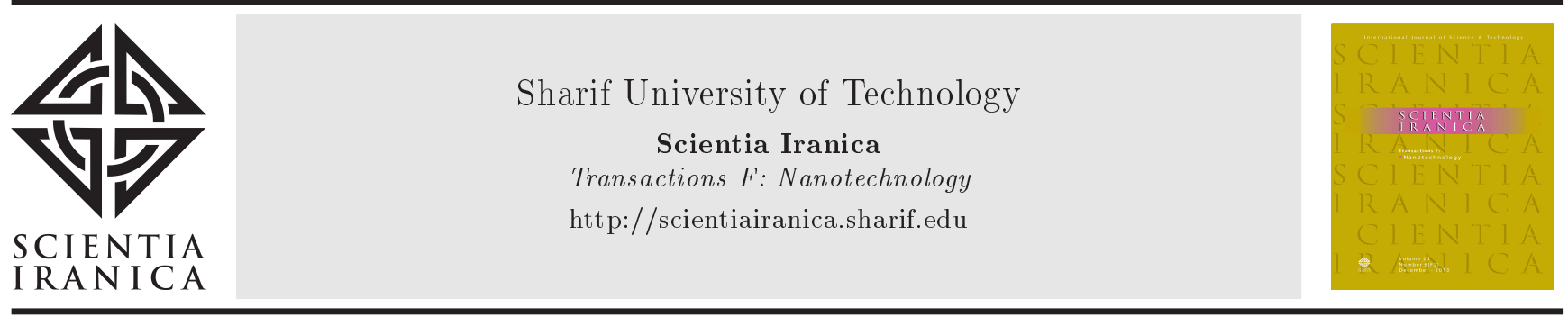

\title{
Modeling for radiated Marangoni convection flow of magneto-nanoliquid subject to activation energy and chemical reaction
}

\author{
I. Ullah ${ }^{\mathrm{a}, *}$, T. Hayat ${ }^{\mathrm{a}, \mathrm{b}}$, A. Alsaedi ${ }^{\mathrm{b}}$, and S. Asghar ${ }^{\mathrm{c}}$ \\ a. Department of Mathematics, Quaid-I-Azam University 45320, Islamabad 44000, Pakistan. \\ b. Nonlinear Analysis and Applied Mathematics (NAAM) Research Group, Department of Mathematics, Faculty of Science, King \\ Abdulaziz University, P. O. Box 80203, Jeddah 21589, Saudi Arabia. \\ c. Department of Mathematics, CUT, Chak Shahzad, Park Road, Islamabad, Pakistan.
}

Received 18 February 2019; received in revised form 29 December 2019; accepted 3 October 2020

\section{KEYWORDS}

Marangoni convection; ESHS;

Activation energy;

Thermal radiation;

Nanomaterials.

\begin{abstract}
Simultaneous impacts of non-linear radiation and magnetohydrodynamics in Marangoni convection nanoliquid, as well as novel aspects of activation energy and space dependent heat source are addressed. Nanoliquid attributes include Brownian movement and thermophoresis diffusion. An NDSolve base shooting technique is employed for the numerical simulation. Aspects of various embedded variables are focused on velocity, heat and mass transport distributions via graphical interpretations, and temperature gradient at the surface is estimated and analyzed. This study identified that the Exponential based Space Heat Source (ESHS) parameter significantly enhanced the thermal field. Activation energy and temperature difference parameters decrease the nanoparticles concentration. Also, temperature gradient enhances for higher Marangoni ratio parameter, Hartmann number, dimensionless activation energy and thermophoresis parameter.
\end{abstract}

C) 2020 Sharif University of Technology. All rights reserved.

\section{Introduction}

Nanoliquid at present is a busy topic for researchers. Choi [1] proposed a novel idea of the suspension of nanoparticles (dimensions less than $100 \mathrm{~nm}$ ) in traditional liquids. He discovered that such a kind of suspension of solid particles in conventional liquids leads to thermal conductivity enhancement. There are several kinds of base liquids namely bio-liquids, lubricants, oils, water, polymer solutions, ethylene glycol etc. Such liquids have useful applications in transformer cooling, electronic cooling and heat ex-

*. Corresponding author. Tel.: +92-5190642172 E-mail address: ikramullah@math.qau.edu.pk (I. Ullah)

doi: $10.24200 /$ sci.2020.52978.2981 changesr [1-3]. Thus, for intensification of thermal potential, fine metallic particles $\left(\mathrm{TiO}_{2}, \mathrm{Cu}, \mathrm{Ag}, \mathrm{Al}_{2} \mathrm{O}_{3}\right.$, Fe and their oxides) are dispersed homogeneously in the operating liquids. Sizeable information about nanoliquids with diverse features is available. Some representative attempts regarding nano-materials can be observed via [4-18]. On the other hand, biodegradability, long blood retention time and the low toxicity of magnetic nanoparticles has become a principal material in biomedical usage. Distinct characteristics like coercivity, high magnetic susceptibility, a large surface to volume ratio, superparamagnetism and low Curie temperature have raised the attention of scientists in magneto-nanoliquids. Nanoparticles composed of magnetic iron oxide are widespread in nature, chemically and physically stable, inexpensive to produce, biocompatible and environmentally safe [19]. Mag- 
netite, hematite and maghemite are typical examples of these materials. Numerous engineering applications regarding magnetic materials include elimination of tumors, asthma treatment, magnetic hyperthermia, drug release, thermoblation, Magnetic Resonance Imaging (MRI), targeted drug delivery, synergistic effects and biosensing (biomolecules and cells). Generally, magnetized nanoparticles exhibit random motion inside the base liquid which would be converted into uniform motion by employing an external magnetic field [20] . Coated magnetized nanoparticles dispersed in anticancer drugs can be injected into a patient's body and movement of the drug to the target region is restrained via a magnetic field [21]. Some analysis in this regard may be noticed in the literature [22-25].

The layers which may arise along the liquidliquid or liquid-gas interactions are referred to as Marangoni layers. Marangoni flow can be both temperature and concentration gradients which are created due to the surface tension gradient. Analysis of Marangoni convection remains an area of high curiosity for engineers and scientists because of its appearance in several practical applications, namely; aerospace, crystal growth, materials science, welding, spreading of thin films, semiconductor processing, nuclear reactors etc. Napolitano [26,27] provided the basic work in this direction and, subsequently, numerous researchers comprehensively scrutinized Marangoni flow with various aspects [28-32]. Xu and Chen [33] explored the heat transport features of Marangoni flow in a copperwater nanoliquid. Sheikholeslami and Chamkha [34] examined aspects of magnetohydrodynamics (MHD) on nanoliquid by considering the Marangoni convection. Thermal Marangoni convective flow of nanoliquid using a rotating disk with irregular heat and solar radiation has been studied by Mahanthesh et al. [35].

The energy that escalates from a heated region to its absorption region in every direction, such as electromagnetic waves is referred to as thermal radiation. It is created by the thermal tumult of composite atoms of the body. Light-bulbs, fire, heat from the microwave and sun radiation are typical examples of thermal radiation. The infrared regime of the electromagnetic spectrum incorporates radiation for most objects on this earth. For this reason, the inspection of radiation is important in the conversation of thermal frameworks. It plays a remarkable role at different high temperature procedures. This concept is also widespread in gas turbines, furnaces, engine cooling, aircraft and boilers etc. Further technological uses of it can be found in solar technology, nuclear power plants, technology related to power, combustion chambers and chemical processes. It has been remarked that aspects of thermal radiation become significant when the difference between ambient and surface temperature is large [3640].
The objective here is to disclose the features of solutal-thermo Marangoni convection in the flow of nanomaterials. Formulation is based upon conservation laws. Attributes of thermal radiation, Brownian motion and thermophoretic diffusion are described. In addition, the impacts of activation energy are examined. Transformations are used to convert flow expressions into ordinary ones. An NDSolve based shooting technique is implemented for the solutions. Plots are shown to describe the behaviors of physical variables, and the Nusselt number is estimated.

\section{Problem formulation}

Radiative Marangoni convective flow of magnetonanoliquid is addressed. A magnetic field of strength, $B_{0}$, is implemented in a $y$-direction (see Figure 1 ). Varying temperature with power law is assumed at the surface. Heat and mass transport is subject to an irregular heat source, thermal radiation and activation energy. Aspects of thermophoretic diffusion and Brownian motion are considered. No relative movement exists between the base liquid and nanoparticles. Moreover, the nanoparticles flux condition is intended. The modeled equations are [28-31,41-45]:

$$
\begin{aligned}
& \frac{\partial u}{\partial x}+\frac{\partial v}{\partial y}=0 \\
& u \frac{\partial u}{\partial x}+v \frac{\partial u}{\partial y}=\nu \frac{\partial^{2} u}{\partial y^{2}}-\frac{\sigma_{1} B_{0}^{2}}{\rho_{f}} u, \\
& u \frac{\partial T}{\partial y}+v \frac{\partial T}{\partial y}=\alpha_{f} \frac{\partial^{2} T}{\partial y^{2}}+\tau\left(D_{B} \frac{\partial C}{\partial y} \frac{\partial T}{\partial y}+\frac{D_{T}}{T_{0}}\left(\frac{\partial T}{\partial y}\right)^{2}\right) \\
& +\frac{Q_{0}\left(T-T_{\infty}\right)}{\left(\rho c_{p}\right)_{f}} e^{-y n \sqrt{\frac{a}{\nu}}}-\frac{1}{\left(\rho c_{p}\right)_{f}} \frac{\partial q_{r}}{\partial y} \\
& u \frac{\partial C}{\partial y}+v \frac{\partial C}{\partial z}=D_{B} \frac{\partial^{2} C}{\partial y^{2}}+\frac{D_{T}}{T_{0}} \frac{\partial^{2} T}{\partial y^{2}}
\end{aligned}
$$

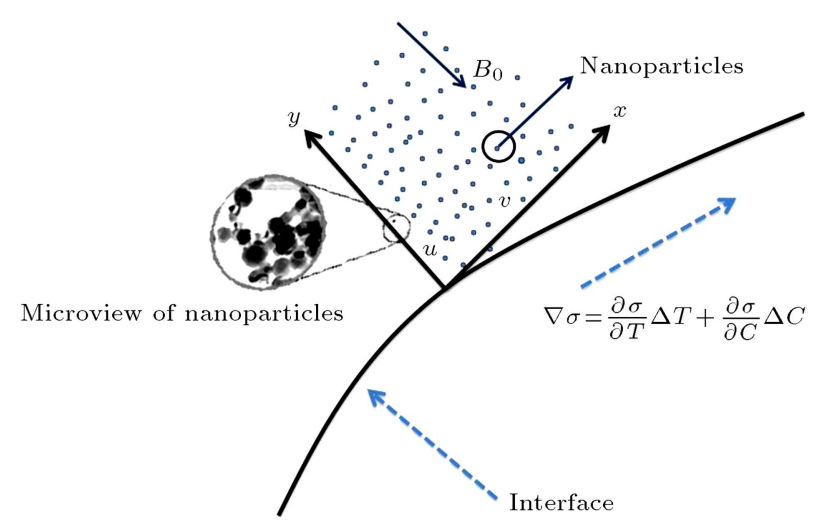

Figure 1. Flow physical schematic diagram. 


$$
-k_{r}^{2}\left(C-C_{\infty}\right)\left(\frac{T}{T_{0}}\right)^{m} \exp \left(\frac{-E_{a}}{\kappa T}\right)
$$

with $[46,47]$ :

$$
\begin{aligned}
& \left.\mu \frac{\partial u}{\partial y}\right|_{y=0}=-\left.\frac{\partial \sigma}{\partial x}\right|_{y=0}=\sigma_{0}\left(\left.\gamma_{C} \frac{\partial C}{\partial x}\right|_{y=0}+\left.\gamma_{T} \frac{\partial T}{\partial x}\right|_{y=0}\right) \\
& v(x, 0)=0, \quad T_{w}(x, 0)=T_{\infty}+T_{0} X^{2} \\
& \left.D_{B} \frac{\partial C}{\partial y}\right|_{y=0}+\left.\frac{D_{T}}{T_{0}} \frac{\partial T}{\partial x}\right|_{y=0}=0 \\
& u(x, \infty) \rightarrow 0, \quad T(x, \infty) \rightarrow T_{\infty}, \quad C(x, \infty) \rightarrow C_{\infty}
\end{aligned}
$$

Here, velocity components parallel to $(x, y)$ are signified by $(u, v), \nu$ the kinematic viscosity, $n$ the exponential index, $\alpha_{f}$ the nanomaterials thermal diffusivity, $\rho_{f}$ the liquid density, $Q_{0}$ heat generation/absorption variable, $\sigma_{1}$ the electrical conductivity, $\tau=\frac{(\rho c)_{p}}{(\rho c)_{f}}$ the heat capacity ratio, $E_{a}$ the non-dimensional activation energy, $D_{B}$ the diffusion coefficient, $D_{T}$ the coefficient of thermophoretic diffusion, $(T, C)$ and $\left(T_{\infty}, C_{\infty}\right)$ the respective ambient and nanoparticles temperature and concentration. The expression for surface tension $(\sigma)$ is:

$$
\begin{aligned}
& \sigma=\sigma_{0}\left[1-\gamma_{C}\left(C-C_{\infty}\right)-\gamma_{T}\left(T-T_{\infty}\right)\right], \\
& \gamma_{C}=-\left.\frac{1}{\sigma_{0}} \frac{\partial \sigma}{\partial C}\right|_{C}, \quad \gamma_{T}=-\left.\frac{1}{\sigma_{0}} \frac{\partial \sigma}{\partial T}\right|_{T},
\end{aligned}
$$

where $\gamma_{T}$ and $\gamma_{C}$ designate the coefficients of temperature and concentration surface tension and $\sigma_{0}$ is a positive constant. Further, in Eq. (4) $\kappa=8.61 \times$ $10(\mathrm{eV} / \mathrm{K})$ is the Boltzmann constant, $m(-1<m>1)$ the fitted rate constant, and $k_{r}^{2}$ the rate of reaction. Radiative heat flux $q_{r}$ is:

$$
q_{r}=-\frac{4 \sigma^{* *}}{3 m^{* *}} \frac{\partial\left(T^{4}\right)}{\partial y}=-\frac{16 \sigma^{* *} T_{\infty}^{3}}{3 m^{* *}} \frac{\partial^{2} T}{\partial y^{2}},
$$

where $m^{* *}$ the coefficient of mean absorption and $\sigma^{* *}$ indicates the Stefan-Boltzman. On using Eq. (8), Eq. (3) yields:

$$
\begin{aligned}
u \frac{\partial T}{\partial y} & +v \frac{\partial T}{\partial y}=\alpha_{f} \frac{\partial^{2} T}{\partial y^{2}}+\tau\left(D_{B} \frac{\partial C}{\partial y} \frac{\partial T}{\partial y}+\frac{D_{T}}{T_{0}}\left(\frac{\partial T}{\partial y}\right)^{2}\right) \\
& +\frac{Q_{0}\left(T-T_{\infty}\right)}{(\rho c)_{f}} e^{-y n_{1} \sqrt{\frac{a}{\nu}}}+\frac{1}{(\rho c)_{f}} \\
& \frac{16 \sigma^{* *} T_{\infty}^{3}}{3 m^{* *}} \frac{\partial^{2} T}{\partial y^{2}} .
\end{aligned}
$$

Setting [47]:

$$
\begin{array}{lrl}
u=\frac{\partial \psi}{\partial y}, & v=-\frac{\partial \psi}{\partial x}, & \psi=v X f(\eta), \\
\eta & =y / L, \quad X=x / L, & T=T_{\infty}+T_{0} X^{2} \theta(\eta), \\
C & =C_{\infty}+C_{0} X^{2} \phi(\eta),
\end{array}
$$

expression (1) is verified trivially while other equations and boundary conditions are:

$$
\begin{aligned}
& f^{\prime \prime \prime}-f^{\prime 2}+f f^{\prime \prime}-\mathrm{Ha} f^{\prime}=0, \\
& (1+R d) \theta^{\prime \prime}+\operatorname{Pr}\left(f \theta^{\prime}-2 f^{\prime} \theta\right)+N_{b} \theta^{\prime} \phi^{\prime}+N_{t} \theta^{\prime 2} \\
& +\operatorname{Pr} Q \exp (-n \eta)=0, \\
& \phi^{\prime \prime}+\operatorname{Sc}\left(f \phi^{\prime}-2 f^{\prime} \phi\right)+\frac{N_{t}}{N_{b}} \theta^{\prime \prime} \\
& -\mathrm{Sc} \alpha_{1}(1+\delta \theta)^{m} \phi \exp \left(\frac{-E}{1+\delta \phi}\right)=0, \\
& f^{\prime \prime}(0)=-2(1+r), \quad f(0)=0, \quad \theta(0)=1, \\
& \phi(0)+\frac{N_{t}}{N_{b}} \theta^{\prime}(0)=0, \quad f^{\prime}(\infty) \rightarrow 0, \quad \theta(\infty) \rightarrow 0, \\
& \phi(\infty) \rightarrow 0
\end{aligned}
$$

where Ha denotes Hartmann number, $N_{b}$ the Brownian motion parameter, $\operatorname{Pr}$ the Prandtl number, $n$ the exponential index, $Q$ the ESHS variable, $N_{t}$ the thermophoresis parameter, Sc the Schmidt number, $\alpha_{1}$ the reaction rate, $L$ the reference length, $R d$ the radiation variable, $\delta$ the temperature difference parameter, $r$ the Marangoni ratio parameter, $E$ the dimensionless activation energy, Ma the solutal, and $\mathrm{Ma}_{T}$ the thermal Marangoni numbers. These variables are quantified by:

$$
\begin{aligned}
& r=\frac{\mathrm{Ma}_{C}}{\mathrm{Ma}_{T}}=\frac{C_{0} \gamma_{C}}{T_{0} \gamma_{T}}, \quad \mathrm{Ma}_{C}=\frac{\sigma_{0} C_{0} \gamma_{C} L}{\alpha \mu}, \\
& \mathrm{Ma}_{T}=\frac{\sigma_{0} T_{0} \gamma_{T} L}{\alpha \mu}, \quad N_{t}=\frac{(\rho c)_{p}}{(\rho c)_{f}} \frac{D_{T} T_{0} X^{2}}{v}, \\
& N_{b}=\frac{(\rho c)_{p}}{(\rho c)_{f}} \frac{D_{B} C_{0} X^{2}}{v}, \quad Q=\frac{Q_{0} L^{2}}{v\left(\rho c_{p}\right)_{f}}, \\
& L=-\frac{\mu v}{\sigma_{0} T_{0} \gamma_{T}}, \quad \operatorname{Pr}=\frac{v}{\alpha_{f}}, \quad \mathrm{Ha}=B_{0} L \sqrt{\sigma_{1} / \mu}, \\
& \mathrm{Sc}=\frac{v}{D_{B}}, \quad \alpha_{1}=L^{2} k_{r}^{2} / v, \quad E=\frac{E_{a}}{\kappa T_{\infty}}, \\
& \delta=\frac{T_{0} X^{2}}{T_{\infty}}, \quad R d=\frac{16 \sigma^{* *} T_{\infty}^{3}}{3 k m^{* *}} .
\end{aligned}
$$




\subsection{Physical quantity}

Local gradient of temperature $\left(\mathrm{Nu}_{x}\right)$ is defined as:

$$
\mathrm{Nu}_{x}=\frac{q_{w} x}{k_{f}\left(T-T_{\infty}\right)}
$$

where:

$$
q_{w}=-\left(k_{f} \frac{\partial T}{\partial y}-\frac{16 \sigma^{* *} T_{\infty}^{3}}{3 m^{* *}} \frac{\partial T}{\partial y}\right)_{y=0} .
$$

In dimensionless form, one has:

$$
\mathrm{Nu}_{x}=-\frac{x}{L}(1+R d) \theta^{\prime}(0)
$$

\subsection{Computational method}

The solutions of Eqs. (12)-(15) are computed numerically by adopting the NDSolve based shooting technique. A well-known computer software, Mathematica, is utilized for the simulation of non-linear problems.

\section{Discussion}

Using the numerical technique highlighted in the aformentioned section, interpretations have been carried out for several values of embedded variables, like Hartmann number $\mathrm{Ha}$, Brownian motion parameter $N_{b}$, Prandtl number $\operatorname{Pr}$, ESHS variable $Q$, Schmidt number Sc, thermophoresis variable $N_{t}$, reaction rate $\alpha_{1}$, radiation parameter $R d$, temperature difference parameter $\delta$, Marangoni ratio parameter $r$, dimensionless activation energy $E$ on non-dimensional velocity $f^{\prime}(\eta)$, temperature $\theta(\eta)$, and concentration $\phi(\eta)$. The involved variables have fixed values:

$$
\begin{aligned}
& n=0.2, \quad \operatorname{Pr}=0.7=\mathrm{Sc}, \quad \delta=0.3=N_{b}, \quad N_{t}=0.1, \\
& \alpha_{1}=R d=0.2=Q=\mathrm{Ha}, \quad m=1.2=r, \quad E=3.0 .
\end{aligned}
$$

In Figure 2 the impact of $\mathrm{Ha}$ on $f^{\prime}(\eta)$ is designed. Here, it was noticed that velocity decays for higher Ha. For Lorentz force increments, a back flow appears

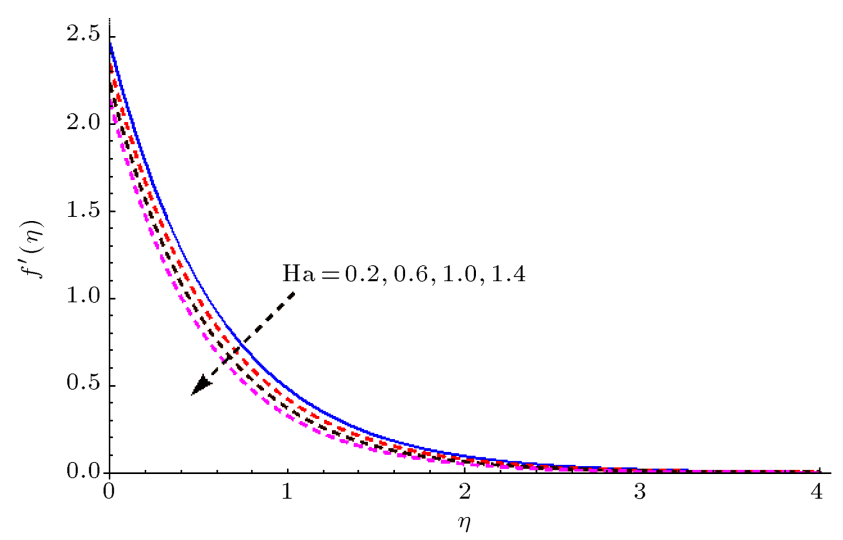

Figure 2. Behavior of $f^{\prime}(\eta)$ via Ha.

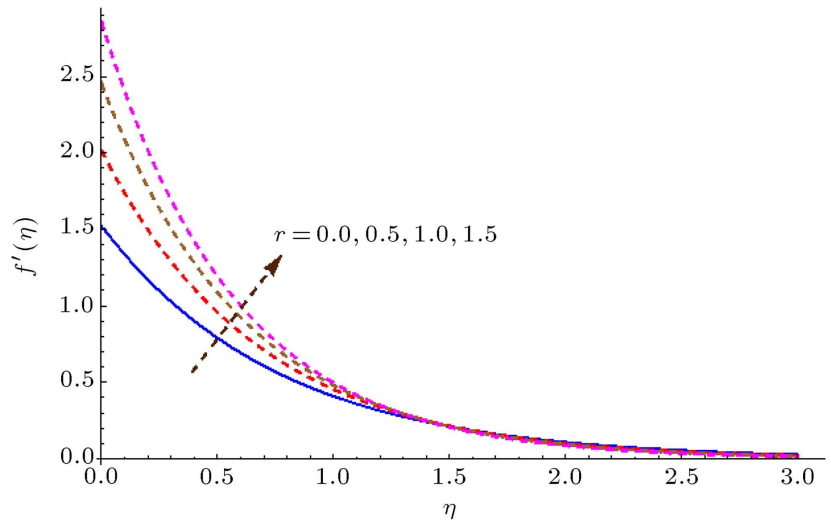

Figure 3. Feature of $f^{\prime}(\eta)$ via $r$.

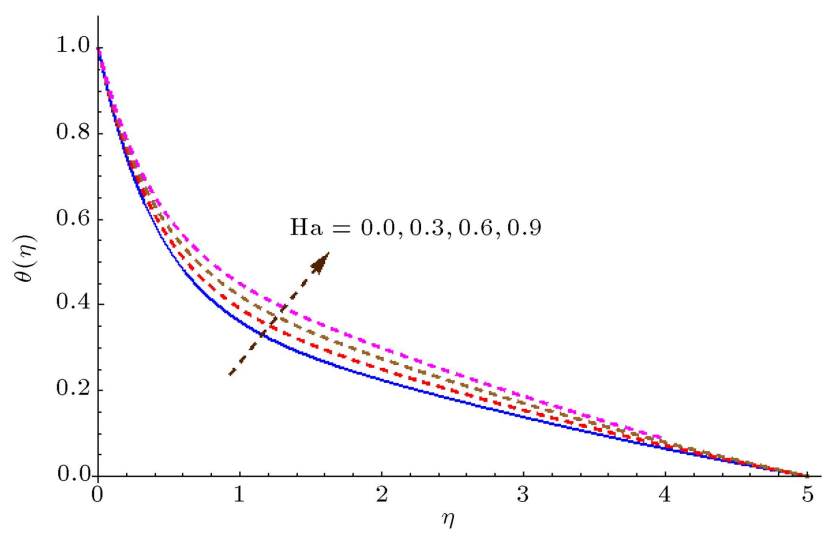

Figure 4. Behavior of $\theta(\eta)$ via Ha.

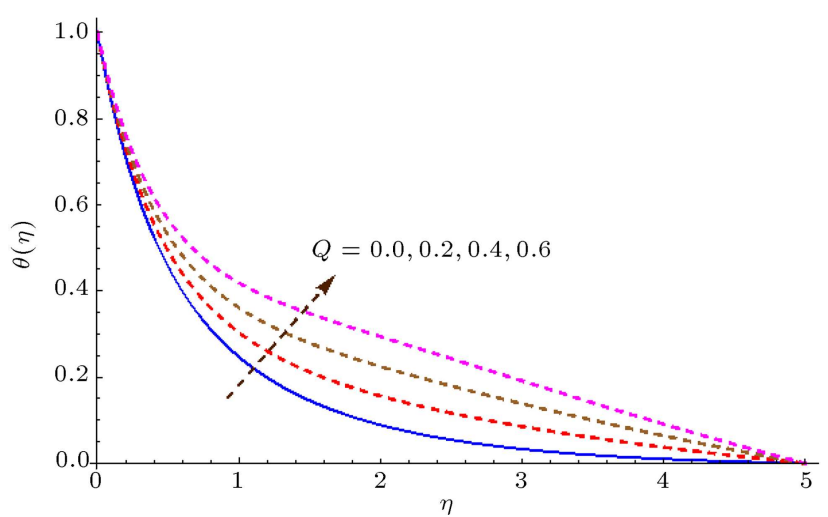

Figure 5. Behavior of $\theta(\eta)$ via $Q$.

and velocity $f^{\prime}(\eta)$ reduces. The thickness of the momentum layer is also reduced. The effect of $r$ on velocity $f^{\prime}(\eta)$ is reported in Figure 3. Clearly, higher estimations of $r$ correspond to more velocity. The influence of $\mathrm{Ha}$ on temperature $\theta(\eta)$ is elucidated in Figure 4. It is found that larger $\mathrm{Ha}$ augment the nanoliquid $\theta(\eta)$. Physical strength in the magnetic field corresponds to an enhanced Lorentz force. This force has a property to endure the liquid movement and thus rise $\theta(\eta)$. Moreover, the situation of hydrodynamics is recovered for $\mathrm{Ha}=0$. Figure 5 is 


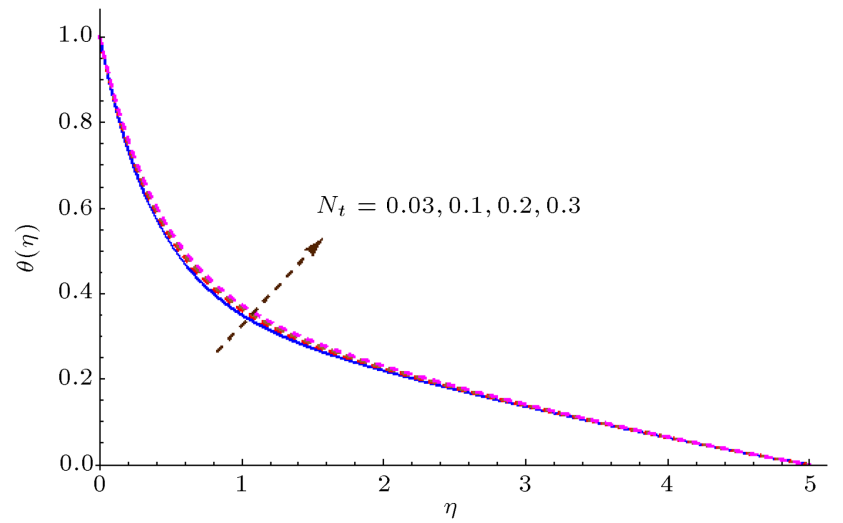

Figure 6. Behavior of $\theta(\eta)$ via $N_{t}$.

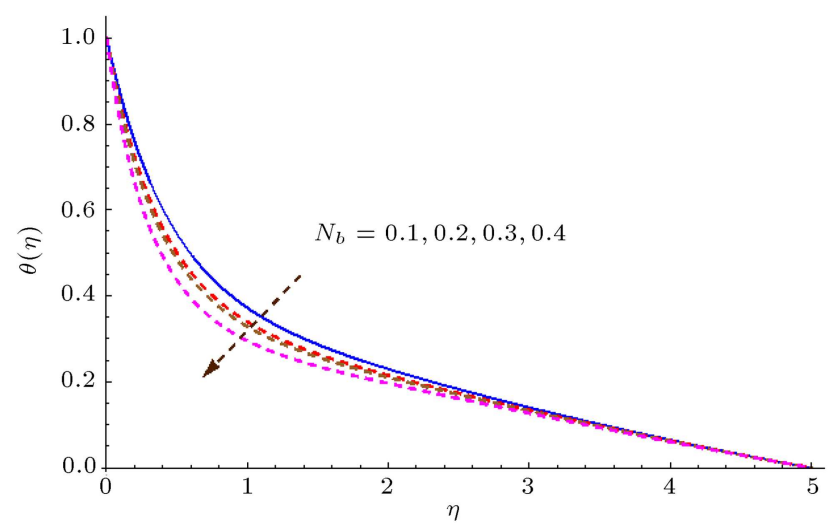

Figure 7. Behavior of $\theta(\eta)$ via $N_{b}$.

interpreted to see changes in $\theta(\eta)$ via $Q$. It is concluded that higher estimations of $Q$ significantly augment the temperature. In fact more heat is produced because of the heat generation procedure within the considered liquid. Interestingly, the thermal field is enhanced significantly for very small estimations, i.e ( 0.0 to 0.6$)$ in the process of ESHS parameters. The importance of thermophoresis parameter $N_{t}$ on $\theta(\eta)$ is captured in Figure 6. Here, the thermal field is improved for larger $N_{t}$. Physically, $N_{t}$ assists thermal diffusion. It means that large numbers of nanoparticles are shifted towards ambient liquid and thus uplift the thermal field and its layer thickness. Figure 7 shows the behavior of $N_{b}$ on $\theta(\eta)$. Here, temperature is the decaying function of $N_{b}$. Figure 8 displays the consequences of $\operatorname{Pr}$ on $\theta(\eta)$. As expected, an increment in $\operatorname{Pr}$ declines thermal diffusion and increases the thermal capacity of liquid, as $\operatorname{Pr}$ has an inverse link with thermal diffusion. Therefore, the strength in Pr declined thermal diffusion, consequently, dropping the temperature. Figure 9 discloses the feature of $r$ on temperature $\theta(\eta)$. Here, $\theta(\eta)$ is reduced for higher estimations of $r$. An enhancement in $R d$ leads to augment temperature distribution. This result is depicted in Figure 10. Kinetic energy progressively enhances due to an increment in $R d$, which makes

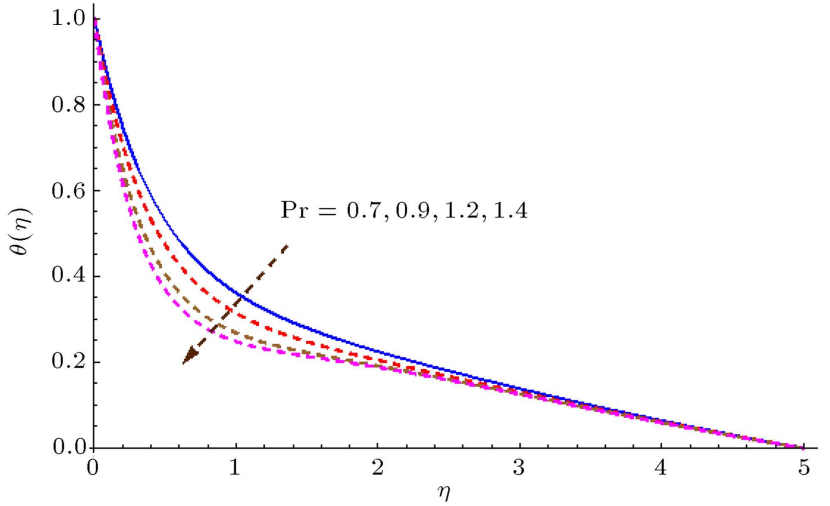

Figure 8. Behavior of $\theta(\eta)$ via $\operatorname{Pr}$.

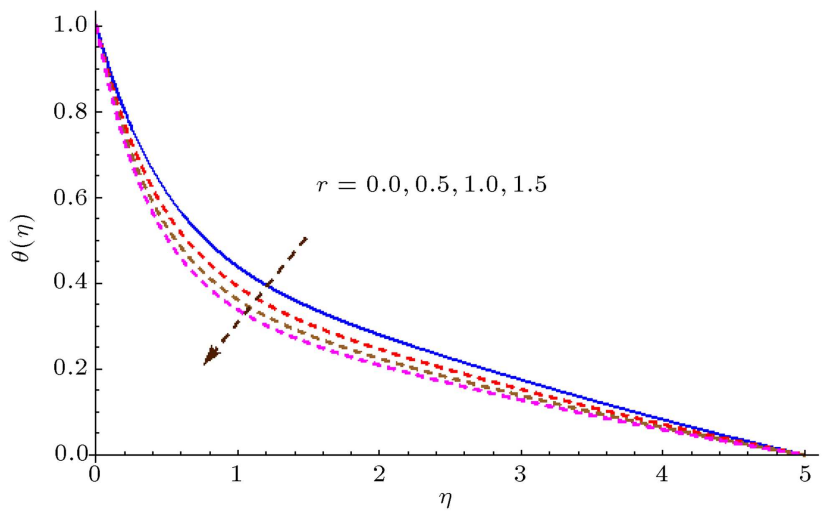

Figure 9. Behavior of $\theta(\eta)$ via $r$.

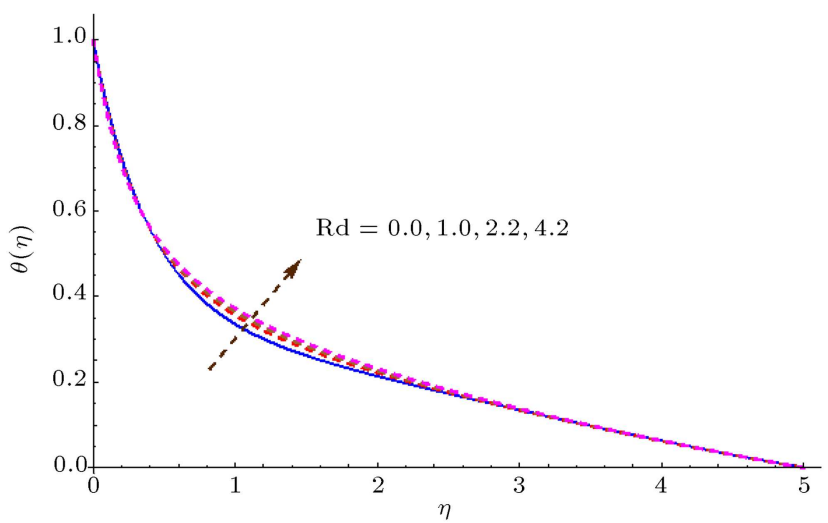

Figure 10. Behavior of $\theta(\eta)$ via $R d$.

the thermal layer thicker. Salient characteristics of $N_{t}$ on concentration field $\phi(\eta)$ are reported in Figure 11. The phenomenon of thermophoresis corresponds to dissemination of the nanoparticles towards ambient liquid from the hot region (as from heated surfaces more resistance is offered to the nanoparticles). Consequently, the thermophoretic force allows nanoparticles to transport heat from the surface to the moving liquids and so $\phi(\eta)$ enhances. The feature of $N_{b}$ on concentration distribution is declared in Figure 12. Here, the $\phi(\eta)$ decaying function of $N_{b}$ is enhanced. 


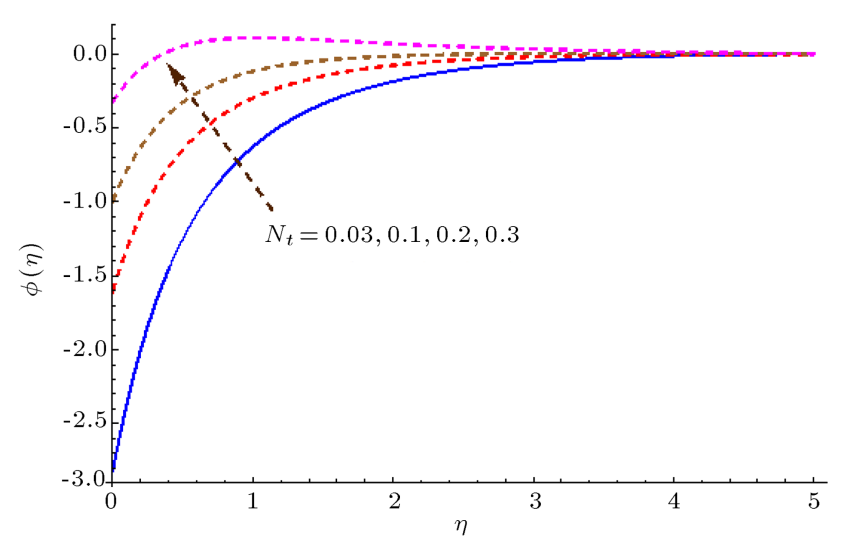

Figure 11. Behavior of $\phi(\eta)$ via $N_{t}$.

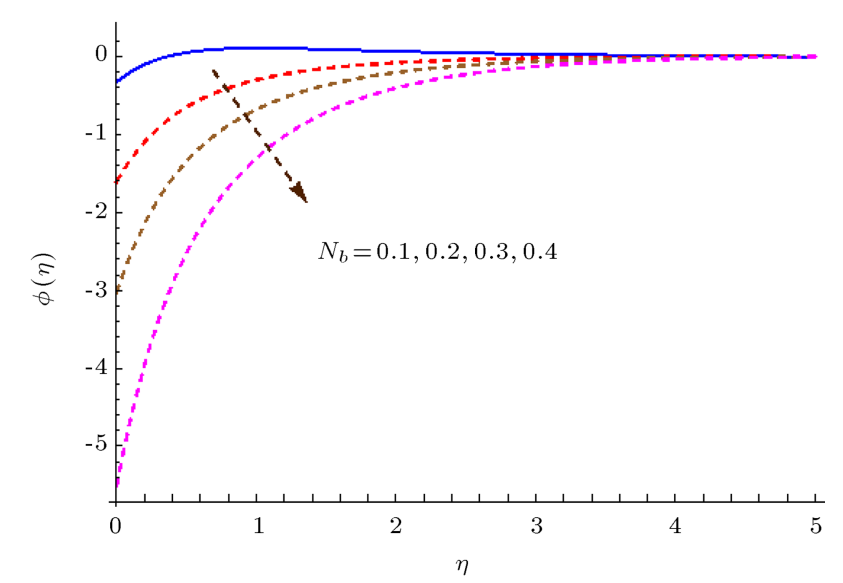

Figure 12. Behavior of $\phi(\eta)$ via $N_{b}$.

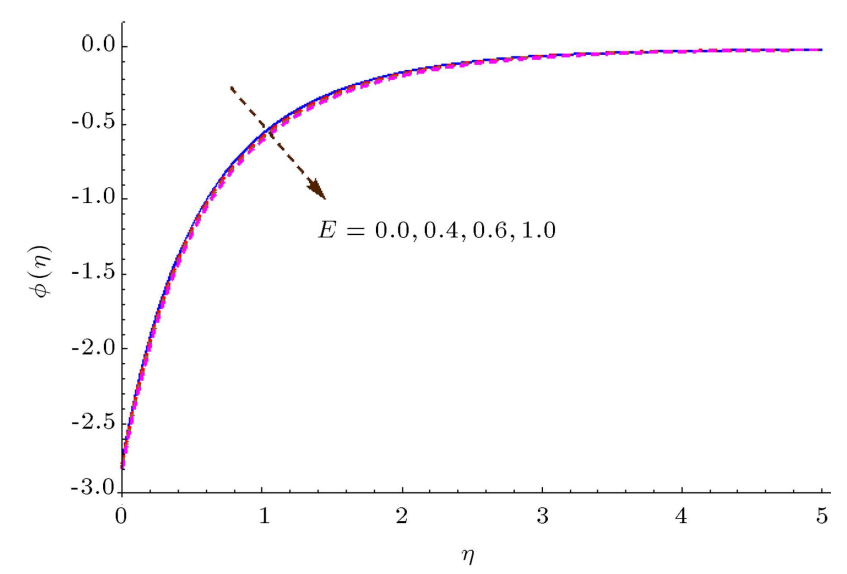

Figure 13. Behavior of $\phi(\eta)$ via $E$.

Physically, Brownian motion occurs due to the correlation of nanoparticles with base liquid in a nanoliquid system. Brownian motion is influenced for higher values of $N_{b}$, which ultimately drops the conduction distribution. Nanoparticle concentration designates a decaying feature for higher activation energy variable (see Figure 13). Figure 14 shows the features of Sc on concentration $\phi(\eta)$. It is found that $\phi(\eta)$ is an increasing function of Sc. The impact of $\alpha_{1}$ on $\phi(\eta)$

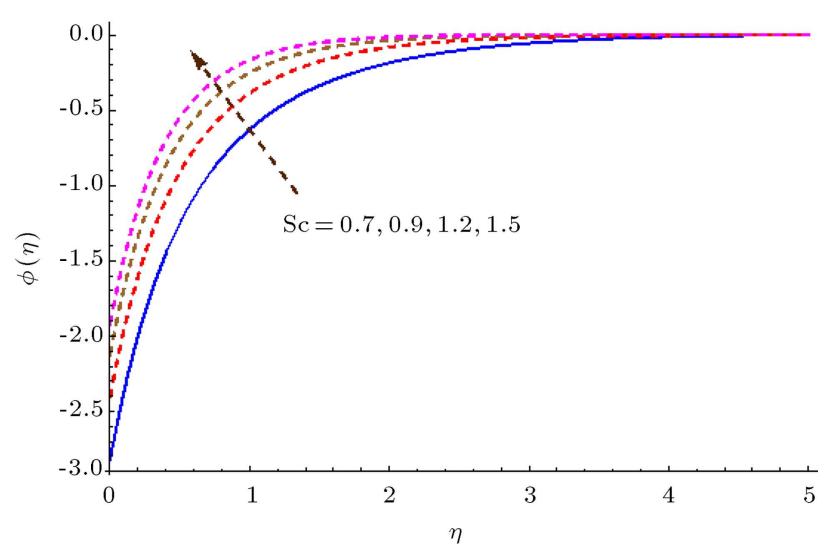

Figure 14. Behavior of $\phi(\eta)$ via Sc.

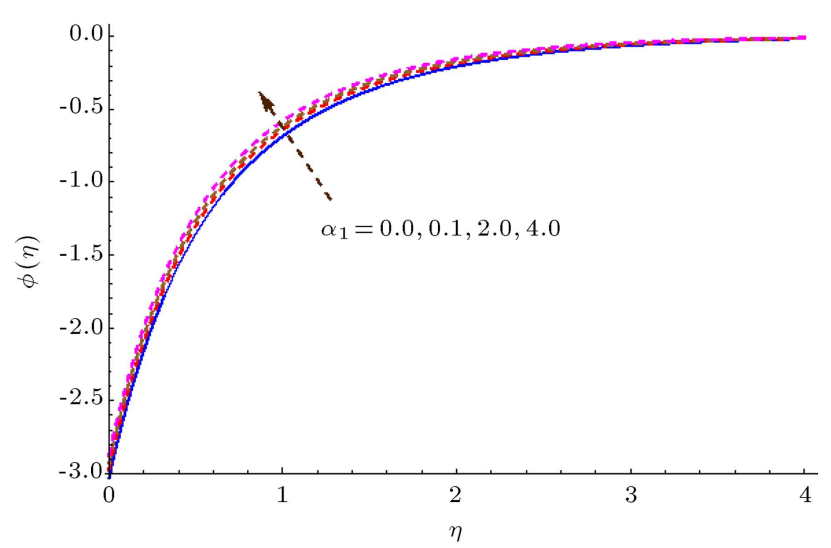

Figure 15. Behavior of $\phi(\eta)$ via $\alpha_{1}$.

is interpreted in Figure 15. Clearly, strength in $\alpha_{1}$ declined $\phi(\eta)$. Aspects of diverse embedding variables on local temperature gradient $\left(\mathrm{Nu}_{x}\right)$ are displayed in Table 1. It is noticed that Nusselt number is enhanced via $\mathrm{Ha}, r, N_{t}$, and $E$.

\section{Concluding remarks}

Magnetohydrodynamic Marangoni convective flow in the presence of activation energy, ESHS and thermal radiation is inspected. Further zero mass flux condition is encountered. Outcomes of the present analysis are summarized as follows:

- Velocities for Marangoni ratio and Hartmann number are opposite;

- Strength in Marangoni ratio on temperature and velocity has a reverse trend;

- Temperature is increased with higher $R d$ and $Q$;

- Features of $N_{b}$ and $N_{t}$ on the thermal field are quite opposite;

- Temperature gradient is enhanced via the Marangoni ratio and activation energy parameters, but a reverse trend is observed for higher heat source parameters; 
Table 1. Numerical estimations of temperature gradient $\left(\mathrm{Nu}_{x}\right)$ for $E, Q$, Ha, $N_{b}, N_{t}$ and $R d$ when $n=0.2, \operatorname{Pr}=0.7=\mathrm{Sc}$, $\delta=0.3=0.1, \alpha_{1}=0.2$, and $m=1.2$.

\begin{tabular}{|c|c|c|c|}
\hline Parameters (fixed values) & Parameters & & $-\theta^{\prime}(0)$ \\
\hline $\begin{array}{c}n=0.2, \operatorname{Pr}=0.7=\mathrm{Sc}, \delta=0.3=N_{b}, m=0.2, \\
N_{t}=0.1, \alpha_{1}=R d=\mathrm{Ha}=Q=0.2, E=3.0\end{array}$ & $r$ & $\begin{array}{l}0.0 \\
1.0 \\
2.0\end{array}$ & $\begin{array}{l}1.00756 \\
1.33393 \\
1.56104\end{array}$ \\
\hline $\begin{array}{c}n=0.2, \operatorname{Pr}=0.7=\mathrm{Sc}, \delta=0.3=N_{b}, m=0.2, \\
N_{t}=0.1, \alpha_{1}=R d=\mathrm{Ha}=Q=0.2, E=3.0, r=1\end{array}$ & $\mathrm{Ha}$ & $\begin{array}{l}0.2 \\
0.5 \\
1.0\end{array}$ & $\begin{array}{l}0.81202 \\
1.27351 \\
1.17967\end{array}$ \\
\hline $\begin{array}{c}n=0.2, \operatorname{Pr}=0.7=\mathrm{Sc}, \delta=0.3, m=0.2, N_{t}=0.1, \\
\alpha_{1}=R d=\mathrm{Ha}=Q=0.2, E=3.0, r=1\end{array}$ & $N_{b}$ & $\begin{array}{l}0.1 \\
0.2 \\
0.3\end{array}$ & $\begin{array}{l}1.27379 \\
1.33393 \\
1.45732\end{array}$ \\
\hline $\begin{array}{c}n=0.2, \operatorname{Pr}=0.7=\mathrm{Sc}, \delta=0.3=N_{b}, m=0.2, \\
\alpha_{1}=R d=\mathrm{Ha}=Q=0.2, E=3.0, r=1\end{array}$ & $N_{t}$ & $\begin{array}{l}0.1 \\
0.3 \\
0.5\end{array}$ & $\begin{array}{l}1.33393 \\
0.66894 \\
0.64616\end{array}$ \\
\hline $\begin{array}{c}n=0.2, \operatorname{Pr}=0.7=\mathrm{Sc}, \delta=0.3=N_{b}, m=0.2 \\
N_{t}=0.1, \alpha_{1}=\mathrm{Ha}=Q=0.2, E=3.0, r=1\end{array}$ & $R d$ & $\begin{array}{l}0.0 \\
0.5 \\
0.9\end{array}$ & $\begin{array}{l}1.57952 \\
1.09983 \\
0.90810\end{array}$ \\
\hline $\begin{array}{c}n=0.2, \operatorname{Pr}=0.7=\mathrm{Sc}, \delta=0.3=N_{b}, m=0.2, \\
N_{t}=0.1, \alpha_{1}=R d=\mathrm{Ha}=0.2, E=3.0, r=1\end{array}$ & $Q$ & $\begin{array}{l}0.0 \\
0.4 \\
0.8\end{array}$ & $\begin{array}{l}1.49673 \\
1.17325 \\
0.85797\end{array}$ \\
\hline $\begin{array}{c}n=0.2, \operatorname{Pr}=0.7=\mathrm{Sc}, \delta=0.3=N_{b}, m=0.2, \\
N_{t}=0.1, \alpha_{1}=R d=\mathrm{Ha}=\mathrm{Q}=0.2, r=1\end{array}$ & $E$ & $\begin{array}{l}0.0 \\
1.0 \\
3.0\end{array}$ & $\begin{array}{l}1.33335 \\
1.33370 \\
1.33393\end{array}$ \\
\hline
\end{tabular}

- Concentration enhances for larger Sc and $\alpha_{1}$.

\section{References}

1. Choi, S.U.S. "Enhancing thermal conductivity of fluids with nanoparticle", ASME Int. Mech. Eng. Cong. Exp., 66, pp. 99-105 (1995).

2. Hu, Z., Lu, W., and Thouless, M.D. "Slip and wear at a corner with Coulomb friction and an interfacial strength", Wear, 338, pp. 242-251 (2015) .

3. Hu, Z., Thouless, M.D., and Lu, W. "Effects of gap size and excitation frequency on the vibrational behavior and wear rate of fuel rods", Nuclear. Eng. Design, 308, pp. 261-268 (2016).

4. Imtiaz, M., Hayat, T., and Alsaedi, A. "Flow of magneto nanofluid by a radiative exponentially stretching surface with dissipation effect", Adv. Powder Technol., 27, pp. 2214-2222 (2016).

5. Turkyilmazoglu, M. "Exact analytical solutions for heat and mass transfer of MHD slip flow in nanofluids", Chem. Eng. Sci., 84, pp. 182-187 (2012).

6. Zheng, L., Zhang, C., Zhang, X., et al. "Flow and radiation heat transfer of a nanofluid over a stretching sheet with velocity slip and temperature jump in porous medium", J. Franklin Inst., 350, pp. 990-1007 (2013).

7. Sheikholeslami, M., Hayat, T., and Alsaedi, A. "On simulation of nanofluid radiation and natural convection in an enclosure with elliptical cylinders", Int. J. Heat Mass Transfer, 115, pp. 981-991 (2017).

8. Hsiao, K. "Stagnation electrical MHD nanofluid mixed convection with slip boundary on a stretching sheet", Appl. Thermal Eng., 98, pp. 850-861 (2016).

9. Shehzad, S.A., Hussain, T., Hayat, T., et al. "Boundary layer flow of third grade nanofluid with Newtonian heating and viscous dissipation", J. Central South Univ., 22, pp. 360-367 (2015).

10. Rahman, S.U., Ellahi, R., Nadeem, S., et al. "Simulta- 
neous effects of nanoparticles and slip on Jeffrey fluid through tapered artery with mild stenosis", J. Mol. Liq., 218, pp. 484-493 (2016).

11. Hayat, T., Ullah, I., Muhammad, T., et al. "Threedimensional flow of Powell-Eyring nanofluid with heat and mass flux boundary conditions", Chin. Phys. B, 25, p. 074701 (2016).

12. Sandeep, N. "Effect of aligned magnetic field on liquid thin film flow of magnetic-nanofluids embedded with graphene nanoparticles", Adv. Powder Techn., 28, pp. $865-875$ (2017).

13. Hayat, T., Muhammad, K., Farooq, M., et al. "Melting heat transfer in stagnation point flow of carbon nanotubes towards variable thickness surface", AIP Advances, 6, p. 015214 (2016).

14. Amala, S. and Mahanthesh, B. "Hybrid nanofluid flow over a vertical rotating plate in the presence of Hall current, nonlinear convection and heat absorption", $J$. Nanofluids, 7, pp. 1138-1148 (2018).

15. Muhammad, T., Lu, D.C., Mahanthesh, B., et al. "Significance of Darcy-Forchheimer porous medium in nanofluid through carbon nanotubes", Commun. Theor. Phys., 70, p. 361 (2018).

16. Shruthy, M. and Mahanthesh, B. "Rayleigh-Bénard convection in Casson and hybrid nanofluids: An analytical investigation", J. Nanofluids, 8, pp. 222-229 (2019).

17. Ashlin, T.S. and Mahanthesh, B. "Exact solution of non-coaxial rotating and non-linear convective flow of $\mathrm{Cu}-\mathrm{Al}_{2} \mathrm{O}_{3}-\mathrm{H}_{2} \mathrm{O}$ hybrid nanofluids over an infinite vertical plate subjected to heat source and radiative heat", J. Nanofluids, 8, pp. 781-794 (2019).

18. Hayat, T., Ullah, I., Alsaedi, A., et al. "Threedimensional mixed convection flow of Sisko nanoliquid", Int. J. Mech. Sci., 133, pp. 273-282 (2017).

19. Si, X., Li, H., Zheng, L., et al. "A mixed convection flow and heat transfer of pseudo-plastic power law nanofluids past a stretching vertical plate", Int. J. Heat Mass Transfer, 105, pp. 350-358 (2017).

20. Khan, W.A., Khan, Z.H., and Haq, R.U. "Flow and heat transfer of ferrofluids over a flat plate with uniform heat flux", Eur. Phys. J. Plus, 130, p. 86 (2015).

21. Wu, W., Wu, Z., Yu, T., et al. "Recent progresses on magnetic iron oxide nanoparticles: synthesis, surface functional strategies and biomedical applications", Sci. Technol. Adv. Mater, 16, p. 023501 (2015).

22. Gireesha, B.J., Gorla, R.S.R., and Mahanthesh, B. "Effect of suspended nanoparticles on threedimensional MHD flow, heat and mass transfer of radiating Eyring-Powell fluid over a stretching sheet", J. Nanofluids, 4, pp. 474-484 (2015).
23. Hayat, T., Ullah, I., Alsaedi, A., et al. "MHD flow of Powell-Eyring nanofluid over a non-linear stretching sheet with variable thickness", Results in Phys., 7, pp. 189-196 (2017).

24. Hayat, T., Saleem, A., Tanveer, A., et al. "Numerical study for MHD peristaltic flow of Williamson nanofluid in an endoscope with partial slip and wall properties", Int. J. Heat Mass Transfer., 114, pp. 1181-1187 (2017).

25. Hayat, T., Ullah, I., Muhammad, T., et al. "Magnetohydrodynamic (MHD) three-dimensional flow of second grade nanofluid by a convectively heated exponentially stretching surface", J. Mol. Liq., 220, pp. 1004-1012 (2016).

26. Napolitano, L.G. "Microgravity fluid dynamics", In 2nd Levitch Conference, Washington (1978).

27. Napolitano, L.G. "Marangoni boundary layers", In Proc. 3rd European Symposium on Material Science in Space, Grenoble, ESA SP-142 (1979).

28. Magyari, E. and Chamkha, A.J. "Exact analytical solutions for thermosolutal Marangoni convection in the presence of heat and mass generation or consumption", Heat Mass Transfer, 43, pp. 965-974 (2007).

29. Lin, L., Zheng, L., and Zhang, X. "MHD Marangoni boundary layer flow and heat transfer of pseudo-plastic nanofluids over a porous medium with a modified model", Mech. Time-Depend. Mater., 19, pp. 519-536 (2015).

30. Hayat, T., Shaheen, U., Shafiq, A., et al. "Marangoni mixed convection flow with Joule heating and nonlinear radiation", AIP Adv., 5, p. 077140 (2015).

31. Aly, E.H. and Ebaid, A. "Exact analysis for the effect of heat transfer on MHD and radiation Marangoni boundary layer nanofluid flow past a surface embedded in a porous medium", J. Mol. Liq., 215, pp. 625-639 (2016).

32. Ellahi, R., Zeeshan, A., and Hassan, M. "Particle shape effects on Marangoni convection boundary layer flow of a nanofluid", Int. J. Numer. Methods Heat Fluid Flow, 26, pp. 2160-2174 (2016).

33. Xu, X. and Chen, S. "Cattaneo-Christov heat flux model for heat transfer of Marangoni boundary layer flow in a copper-water nanofluid", Heat Transfer-Asian Res., 18, pp. 1-13 (2017).

34. Sheikholeslami, M. and Chamkha, A.J. "Influence of Lorentz forces on nanofluid forced convection considering Marangoni convection", J. Mol. Liq., 225, pp. 750-757 (2017).

35. Mahanthesh, B., Gireesha, B.J., Shashikumar, N.S., et al. "Marangoni convective MHD flow of SWCNT and MWCNT nanoliquids due to a disk with solar radiation and irregular heat source", Physica E, 94, pp. 25-30 (2017).

36. Hayat, T., Ullah, I., Alsaedi, A., et al. "Simultaneous effects of non-linear mixed convection and radiative flow due to Riga-plate with double stratification", $J$. Heat Transfer (2018). Doi: 10.1115/1.4039994 
37. Animasaun, I.L., Raju, C.S.K., and Sandeep, N. "Unequal diffusivities case of homogeneous-heterogeneous reactions within viscoelastic fluid flow in the presence of induced magnetic-field and nonlinear thermal radiation", Alex. Eng. J., 55, pp. 1595-1606 (2016).

38. Hayat, T., Haidera, F., Muhammad, T., et al. "DarcyForchheimer squeezed flow of carbon nanotubes with thermal radiation", J. Phys. Chem. Solids, 120, pp. $79-86$ (2018).

39. Hayat, T., Ullah, I., Alsaedi, A., et al. "Radiative flow of Carreau liquid in presence of Newtonian heating and chemical reaction", Results Phys., 7, pp. 715-722 (2017).

40. Hayat, T., Ullah, I., Muhammad, T., et al. "Radiative three-dimensional flow with Soret and Dufour effects", Int. J. Mech. Sci., 133, pp. 829-837 (2017).

41. Gireesha, B.J., Mahanthesh, B., and Prasannakumara, B.C. "Exploration of activation energy and binary chemical reaction effects on nano Casson fluid flow with thermal and exponential space-based heat source", Mult. Mod. Mat. Structures, 15, pp. 227-245 (2019).

42. Hayat, T., Ullah, I., Waqas, M., et al. "Attributes of activation energy and exponential based heat source in flow of Carreau fluid with cross-diffusion effects", J. Non-Equilib. Thermodyn., 44, pp. 203-213 (2018). Doi.10.1515/jnet-2018-0049

43. Hsiao, K.L. "To promote radiation electrical MHD activation energy thermal extrusion manufacturing system efficiency by using Carreau-nanofluid with parameters control method", Energy, 130, pp. 486-499 (2017).

44. Hsiao, K.L. "combined electrical MHD heat transfer thermal extrusion system using Maxwell fluid with radiative and viscous dissipation effects", Appl. Therm. Eng., 112, pp. 1281-1288 (2017).

DOI: $10.1016 /$ j.applthermaleng.2016.08.208

45. Hsiao, K.L. "Micropolar nanofluid flow with MHD and viscous dissipation effects towards a stretching sheet with multimedia feature", Int. J. Heat Mass Transfer, 112, pp. 983-990 (2017).

46. Hayat, T., Ullah, I., Waqas, M., et al. "Flow of chemically reactive magneto Cross nanoliquid with temperature-dependent conductivity", Appl. Nanosci., 8, pp. 1453-1460 (2018).

47. Hayat, T., Ullah, I., Waqas, et al. "Simulation of nanofluid thermal radiation in Marangoni convection flow of non-Newtonian fluid: A revised model", Int. J. Num. Meth. Heat Fluid Flow, 29, pp. 2840-2853 (2019).

\section{Biographies}

Ikram Ullah is a $\mathrm{PhD}$ student of Mathematics at Quaid-i-Azam University, Pakistan. He received his MS and Mphil degrees from Quaid-i-Azam University. His research interests include nanomaterials, hybrid nanomaterials, entropy analysis and heat mass transfer.

Tasawar Hayat is a Pakistani Mathematician who has made pioneering research contributions to the area of mathematical fluid mechanics. He is considered one of the leading mathematicians working in Pakistan and, currently, is Professor of Mathematics at the QuaidiAzam University.

Ahmad Alsaedi is Professor in the Department of Mathematics at King Abdulaziz University, Jeddah, Saudi Arabia. He is a member of the Nonlinear Analysis and Applied Mathematics (NAAM) research group. His areas of interest include fluid dynamics, nonlinear flow analysis and flow problems in nanosystems.

Saleem Asghar is an eminent Professor at Comsats Institute of Information Technology. His research interests are fluid dynamics, wave propagation and engineering. 